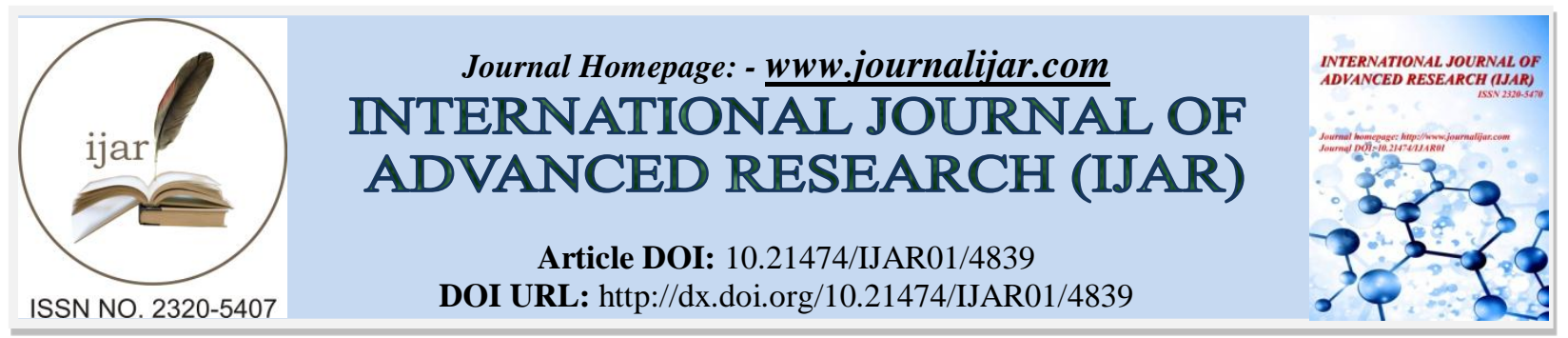

RESEARCH ARTICLE

\title{
SAFETY OF ONCOPLASTIC REDUCTION MAMMOPLASTY: FIVE-YEARS TERTIARY CARE CENTER EXPERIENCE.
}

\footnotetext{
Ali Hadadi, MBBS ${ }^{1,2^{*}}$, Obaid Almeshal, M.D ${ }^{1,2,3}$, Manal Alzaid, M.D ${ }^{1,2,4}$ and Reem Aljehani, M.D ${ }^{1,2,3}$.

1. Researcher, King Abdullah International Medical Research Center, Riyadh, Saudi Arabia.

2. College of Medicine, King Saud bin Abdulaziz University for Health Sciences, Riyadh, Saudi Arabia.

3. Department of Plastic Surgery, King Abdulaziz Medical City, Ministry of National Guard Health Affairs, Riyadh, Saudi Arabia.

4. Department of General Surgery, King Abdulaziz Medical City, Ministry of National Guard Health Affairs, Riyadh, Saudi Arabia.
}

\section{Manuscript Info}

Manuscript History

Received: 13 May 2017

Final Accepted: 15 June 2017

Published: July 2017

Key words:-

Breast Cancer; Mammoplasty;

Oncoplastic Surgery; Safety.

\section{Abstract}

Background: Oncoplastic reduction mammoplasty (ORM) had expanding the role of breast conserving surgery (BCS). However, concerns regarding the oncological safety of this procedure have been expressed. The purpose of this study was to evaluate the safety of (ORM) surgery in King Abdulaziz Medical City (KAMC).

Methods: Breast cancer patients treated with ORM between 2010 and 2015 were included. Patients' demographics and clinical outcomes including, the location and histological characteristic of breast cancer, types of breast reduction techniques, tumor margin positivity and history characteristics were queried.

Results: The study included fifteen patients the mean age was 47 years with SD 9.1. There were no mortalities or major complications other than positive margin after the surgery. Right breast cancer was the most common 67\%. Invasive Ductal Carcinoma (IDC) was the commonest type $80 \%$. The pathological stage ypT1N0Mx was the most common $46 \%$ and the pathological Grade 3 was the most common grade overall $67 \%$. The tumor size range from $4 \mathrm{~mm}$ to 50 $\mathrm{mm}$ with mean $26.41 \mathrm{~mm}$ and SD 17.15. The inferior pedicle breast reduction was the most common technique account for $67 \%$. The majority of patients $(87 \%)$ did not require any further breast surgery. However, two patients (13\%) of all cases required further breast surgery after the ORM.

Conclusions: Our experience with ORM showed that it is a safe and reliable procedure with better cosmetic outcome in our population. Further multicenter study including larger sample size in Saudi Arabia is necessary.

Copy Right, IJAR, 2017,. All rights reserved.

\section{Introduction:-}

The surgical intervention for breast cancer is evolving throughout the centuries. In The Smith Surgical Papyrus 
(3000-2500 b.c.), the author concluded with "There is no treatment." [1]. However, with recent advances in the surgical intervention nowadays, there is a concern about excising the breast cancer with best possible cosmetic results. This surgical intervention called "oncoplastic" which is a Greek word literally means "moulding of the tumour". This term was documented in the literature first time at 1997 [2]. The oncoplastic breast surgery techniques were first described by Audretsh, the father of oncoplastic surgery, in 1998 [3]. Since the introduction of oncoplastic breast surgery in 1998, there are radical changes in the surgical intervention of breast cancer. Nowadays, oncoplastic sugery is a central element of the breast multidisciplinary team [4].

Oncoplastic breast conserving surgery (BCS) has the potential to enhance the aesthetic outcome of BCS. In addition, oncoplastic reduction mammoplasty (ORM) had expanding the role of BCS in situations previously considered unsuitable for conservation because of large tumor size, central and lower pole tumor location or multifocality. However, concerns regarding the oncological safety of this procedure have been expressed [5].

Due to the fact that there is no consensus regarding the best approach of ORM and lack of studies in our country Saudi Arabia, this study will be conducted to review our experience with ORM, to establish a clinical pathway of oncoplastic breast surgery patients and to compare our findings to international studies.

\section{Methods:-}

This hospital-based study was carried out over thirteen months period at King Abdulaziz Medical City, a tertiary care center in Riyadh. The study included all ORM cases treated in our institution throughout 5-year period from January 2010 to December 2015. Ethical approval for the study was obtained from the ethics committee of King Abdullah International Medical Research Center (KAIMRC) with research protocol SP 16/021.

A total of 31 breast cancer patients were managed in our institution during 5-year period and by including only the cases that underwent ORM, a total of 15 patients were included and form the basis of the present study.

Data collection included the age of patient, sex, site of breast cancer, breast cancer type, breast cancer stage and grade, tumor size, the molecular marker including estrogen receptor (ER), progesterone receptor (PR) and human epidermal growth factor receptor 2 (HER2), type of breast reduction surgery, sentinel lymph node biopsy, radiotherapy, completion surgery, and breast cancer risk factor including age of menarche, age of fist birth, breast feeding, oral contraceptive pill (OCP) and family history, medical history and surgical history.

To reflect the most possible precise outcome of the present study, the patient's hospital records of clinical examinations, investigations, and/or surgery were the only source used to collect the data.

\section{Surgical Technique:-}

Preoperative planning for all patients was coordinated by the senior plastic surgeon and the senior surgical oncologist. Preoperative mammogram confirmation of single tumor and the clinical impression of negative margin could be achieved without mastectomy was prerequisite for inclusion of patients in this study to maintain the standard criteria for breast conservation surgery. The exclusion criteria were multicentric carcinoma, inflammatory breast cancer, inability to achieve negative surgical margin, contraindication to radiotherapy, small breast size, central tumor, patient's comorbidity and treatment preference.

In all patients, preoperative marking was performed according to site of tumor and preoperative keyhole-pattern skin resection marking were underwent by the senior plastic surgery team. First, the senior surgical oncologist carried out the tumor resection utilizing the keyhole-pattern skin marking. The surgical oncologist performed sentinel lymph node biopsy through injection of radionucleotide tracer preoperatively and isosulfan blue dye injection 3-5 $\mathrm{ml}$ intraoperatively. Second, sentinel lymph node biopsy were performed by the senior surgical oncologist after resection of tumor. Excision of tumor with minimum margin of $1.5 \mathrm{~cm}$ was maintained for all cases. Third, intraoperative frozen section following tumor excision was performed for margin control. We consider positive margin status if it is less than $2 \mathrm{~mm}$ in the pathological evaluation. Finally, after the senior surgical oncologist has resected all the cancerous tissue, the senior plastic surgery team has completed the reconstruction utilizing reduction mammoplasty for the ipsilateral and contralateral breast. The type of pedicle used "superior or inferior "were determined by the tumor location and size. All resected tissue was labeled and send for pathological evaluation. The senior plastic surgeon has evaluated the symmetry of the ipsilateral and contralateral breast on the operating table. The surgeons and the medical oncologist follow up the patients regularly every 2 weeks for the first month, every 3 months for the next 2 years and annually thereafter. 


\section{Statistical Analysis:-}

Means and standard deviations were used to summarize continuous variables. Frequencies and proportions were used to present the categorical clinical characteristics. The $\chi 2$ test (or Fisher exact test) was used to compare data. All tests were two sided and a $\mathrm{P}<0.05$ was considered statistically significant. The Statistical Package for Social Sciences (IBMSPSS, Oklahoma, USA, version 21) was used for data management and analysis.

\section{Results:-}

A total of fifteen breast cancer patients underwent ORM surgery from January 2010 to December 2015. There were no mortalities or major complications other than positive margin after the surgery. The mean age was 47 years with SD 9.1. Right breast cancer was the most common 67\% with left breast cancer account for 33\%. Three different types of breast cancer were identified with (IDC) account for the majority $80 \%$. The other two types are (DCIS) and (ILC) account for 7\% and $13 \%$ respectively. The pathological stage ypT1N0Mx was the most common $46 \%$ followed by T2NOM0 18\%. In addition, the pathological grade 3 (G3) was the most common grade overall $67 \%$. Grade 1 (G1) and grade 2 (G2) accounted for $13 \%$ and $20 \%$ respectively. The tumor size range from $4 \mathrm{~mm}$ to 50 $\mathrm{mm}$ with mean $26.41 \mathrm{~mm}$ and SD 17.15.Table1 illustrate the baseline characteristic of ORM patients.

The history characteristics of all our cases were analyzed including the mean age of menarche 12.71 years with SD 1.85 and the mean age of first childbirth was 21.71 years with SD 3.87. Family history of breast cancer was negative in the majority of cases 9 patients (60\%). Positive family history of breast cancer was reported in 5 patients (33.3\%). Moreover, the majority of patients had breast fed their children 12 patients $(80 \%)$ with only one patient did not do so. Analysis of the molecular markers in breast cancer tissues were considered in our study with (ER) and (PR) were positive in the majority of cases $73 \%$ and $60 \%$ respectively. On the contrary (Her2) was negative in the majority of cases $67 \%$. Although ER and PR were more positive in right breast, these were not statistically significant $(\mathrm{p}=0.409)$ and $(\mathrm{p}=0.264)$ respectively. Only two patients $(13.3 \%)$ had BRCA1 mutation. In addition, the use of OCP was identified in 9 patients $(60 \%)$. Table 2 illustrate the history characteristics of ORM patients.

The median interval from the breast cancer diagnosis to the surgical management by ORM techniques was 51 days with the minimum and maximum interval were one day and 274 days respectively Fig. 1 . In our practice, the inferior pedicle breast reduction was the most common technique account for $67 \%$ followed by the superior pedicle breast reduction 33\% Fig.2. Moreover, the majority of patient had undergone right axillary sentinel lymph node biopsy $(60 \%)$ and $(27 \%)$ of cases had undergone left axillary sentinel lymph node biopsy. Only $13 \%$ of all cases had not undergone sentinel lymph node biopsy. However, the result of the sentinel lymph nodes biopsy was negative for cancer in the majority of the cases $87 \%$.

The majority of patients (87\%) who underwent ORM in our institution were satisfied with this modality of breast cancer management as interviewed in the follow up and did not require any further breast surgery. However, two patients (13\%) of all cases required further breast surgery after the ORM Fig.3. One patient underwent Skin-sparing mastectomy with retropectoral implant and the other patient underwent right axillary lymph node dissection. Of note, both patients who required a further re-excision after ORM had left breast cancer invasive ductal carcinoma "IDC". Our surgical outcomes of inferior pedicle technique and superior pedicle technique are demonstrated in Fig.4 and Fig.5 respectively.

\section{Discussion:-}

ORM has been a reliable technique to manage breast cancer because of its safety, equivalent oncologic outocme to radical mastectomy, low sugical morbidites and high patient satisfaction and functional outcome [6-8] . However, in Saudi Arabia, to our knowledge, there is no single study had investigated the safety of ORM in this population. Therefore, the focus of this study is to assess the safety of ORM in this unique population.

We investigated the safety of ORM surgery in KAMC including all cases from January 2010 to December 2015. The mean age was 47 years with SD 9.1. There were no mortalities or major complications other than positive margin after the surgery. In term of location of breast cancer and type, right breast cancer was the most common $67 \%$ and (IDC) was the commonest type $80 \%$. The pathological stage ypT1N0Mx was the most common $46 \%$ and the pathological Grade 3 was the most common grade overall 67\%. The tumor size range from $4 \mathrm{~mm}$ to $50 \mathrm{~mm}$ with mean $26.41 \mathrm{~mm}$ and SD 17.15. The inferior pedicle breast reduction was the most common technique accounted for 
$67 \%$. In the present study, the majority of patients (87\%) did not require any further breast surgery. However, two patients (13\%) of all cases required further breast surgery after the ORM.

The decision whether a patient is candidate or not for ORM depends on many factors including breast size, tumor location, how dense is the breast gland, tissue excision volume, patient's comorbidity especially diabetes, obesity, smoking and previous surgery, whether or not the patient receive any chemotherapy, radiotherapy or hormonal therapy before the ORM [4].

In the present study, right breast cancer account for the majority of cases (80\%), however, right breast cancer was half of the cases (53.6\%) in study by M. Emiroglu et al. from Turkey [9]. In our study (IDC) account for the majority $80 \%$, followed by (DCIS) and (ILC). Among most of the studies we found that the most common pathology is IDC and most of literature indicate that IDC is the commonest breast pathology [10].

In our study, the tumor size range from $4 \mathrm{~mm}$ to $50 \mathrm{~mm}$ with mean $26.41 \mathrm{~mm}$, which is comparable to the reported mean of $26 \mathrm{~mm}$ with range of $4 \mathrm{~mm}$ to $47 \mathrm{~mm}$ by study from Turkey [9]. These similarities could be explained by the fact that ORM targets patients in the early stage of breast cancer. However, the pathological grade G3 was the commonest in the present study yet G2 was the commonest and G3 was the least common in a study from Turkey [9]. The molecular markers of the present study have shown that the majority of PR and ER are positive and Her2 is mainly negative and this is similar to the reported molecular marker [9].

The history characteristic of ORM patients in the present study were analyzed with 33.3\% positive family history of breast cancer, which is higher than reported rate (12.4\%) [9]. This difference could be explained by the small sample size of the present study compared to that study, 15 patients and 82 patients respectively.

Risk of further positive margin and the need for further operation is an important aspect of ORM that patient in particular, who are young with large tumor size, should be fully aware and appropriately informed about [11]. However, recurrence rate in ORM was comparable to the standard breast conserving surgery with even decrease the necessity of repeat surgery with the former as indicated by one of larger series [6]. ORM is an excellent choice for patients since it does not jeopardize the oncological safety while achieving the best possible aesthetic outcome. The assessment of treatment should report four measurable outcomes, which are the survival rate, patients' satisfaction, aesthetic outcome and local recurrence rate. However, ORM can be time consuming, technically demanding and require specialist training which have cost and resource implication for the institution [6].

Currently, there is growing evidence that oncoplastic techniques offer the patients safe and effective oncological outcomes. Kaur et al found that a larger volume excision is possible in a subset of patients treated by oncoplastic techniques and they found it is superior to standard breast conserving surgery which carry the risk of positive margin in large tumor [12]. Moreover, Fitoussi et al conducted a study of 540 consecutive ORM between 1986 and 2007 revealed a local recurrence rate of $6.8 \%$ and conclude with safety of ORM [7] .

The complication of positive margin after ORM remains a challenge for the surgical team. In our practice, the positive margin after ORM was $13 \%$, which is comparable to the reported rate of positive margin post ORM (12.4\%) by Loksen et al [13]. In addition, this is similar to the reported rate (16\%) of completion mastectomy after the oncoplastic surgery [14]. Moreover, a study conducted by McCulley and MacMillan from United Kingdom revealed the reoperation rate was $8 \%$ due to positive margin post surgery [15].

Notably both patients who required a further re-excision after ORM had left breast cancer (IDC) in the present study, however, this is different from the reported breast cancer type (ILC) that required reoperation [15].

ORM has been shown to decrease the risk of radiation toxicity and reduce the risk of cancer proportionally to the excised breast tissue $[16,17]$. In addition, ORM advantages in patients with macromastia include reduce the upper limb pain and clothing fit improvement [18].

Despite the topic of adjuvant radiotherapy post ORM has been controversial in the literature, currently, most of centers practice the radiotherapy post ORM and agree about its safety [19]. In the present study, all of ORM patients had adjuvant radiotherapy. 
Currently, there is no consensus about a reliable objective tool to assess the aesthetic outcome of ORM. However, the BREAST-Q which is a validated questionnaire that may help increasing the perception of the outcome of ORM through pre and post operative survey that take the patient quality of life and satisfaction into consideration [20]. In the present study, during the follow up, the patients reported 100\% satisfaction of cosmetic result of ORM which is comparable to study by Chang et al. from United States which report 100\% satisfaction with aesthetic and functional outcomes [21]. The majority of our patients had a large breast volume, which is a significant factor to consider ORM. This finding may explain the high satisfaction among our patient because the larger breasts are more likely to withstand the resection without jeopardizing the cosmetic outcome [22].

We acknowledge the limitations of our study. This investigation was conducted in a single institution. Therefore the findings of this study might not be representative of all ORM patients in Saudi Arabia. Moreover, the small sample size is a limiting factor to present the statistical significance of the findings. The objective assessment of patients' satisfaction by using BREAST-Q survey was not fulfilled by this study and we depend solely on patients interview during the follow up.

\section{Conclusion:-}

ORM techniques are increasingly used to facilitate breast conservation and maintain breast aesthetics but evidence with regards to the oncological safety of oncoplastic breast conservation surgery remains limited. Our experience with ORM showed that the creative use of reconstructive techniques can yield excellent results with a minimum rate of morbidity. Therefore, the authors conclude that ORM is a safe and reliable procedure with better cosmetic outcome in Saudi women. However, further multicenter long-term study including larger sample size in Saudi Arabia is necessary to assess the long-term outcome of ORM in Saudi women.

\section{Acknowledgment:-}

We would like to thank King Abdullah International Medical Research Center (KAIMRC) for their support. Also, the authors appreciate the help of Motasim Badri to this study.

\section{Compliance with ethical standards:- \\ Ethical approval:-}

Ethical approval for the study was obtained from the ethics committee of King Abdullah International Medical Research Center (KAIMRC) with research protocol SP 16/021.

\section{Patient Consent:-}

Before the patients' inclusion in this study, informed written consents were taken.

\section{Conflict of interest statement:-}

Ali Hadadi, Obaid Almeshal, Manal Alzaid and Reem Aljehani declare that they have no conflict of interest.

\section{Funding:-}

This research received no specific grant from any funding agency in the public, commercial, or not-for-profit sectors. 


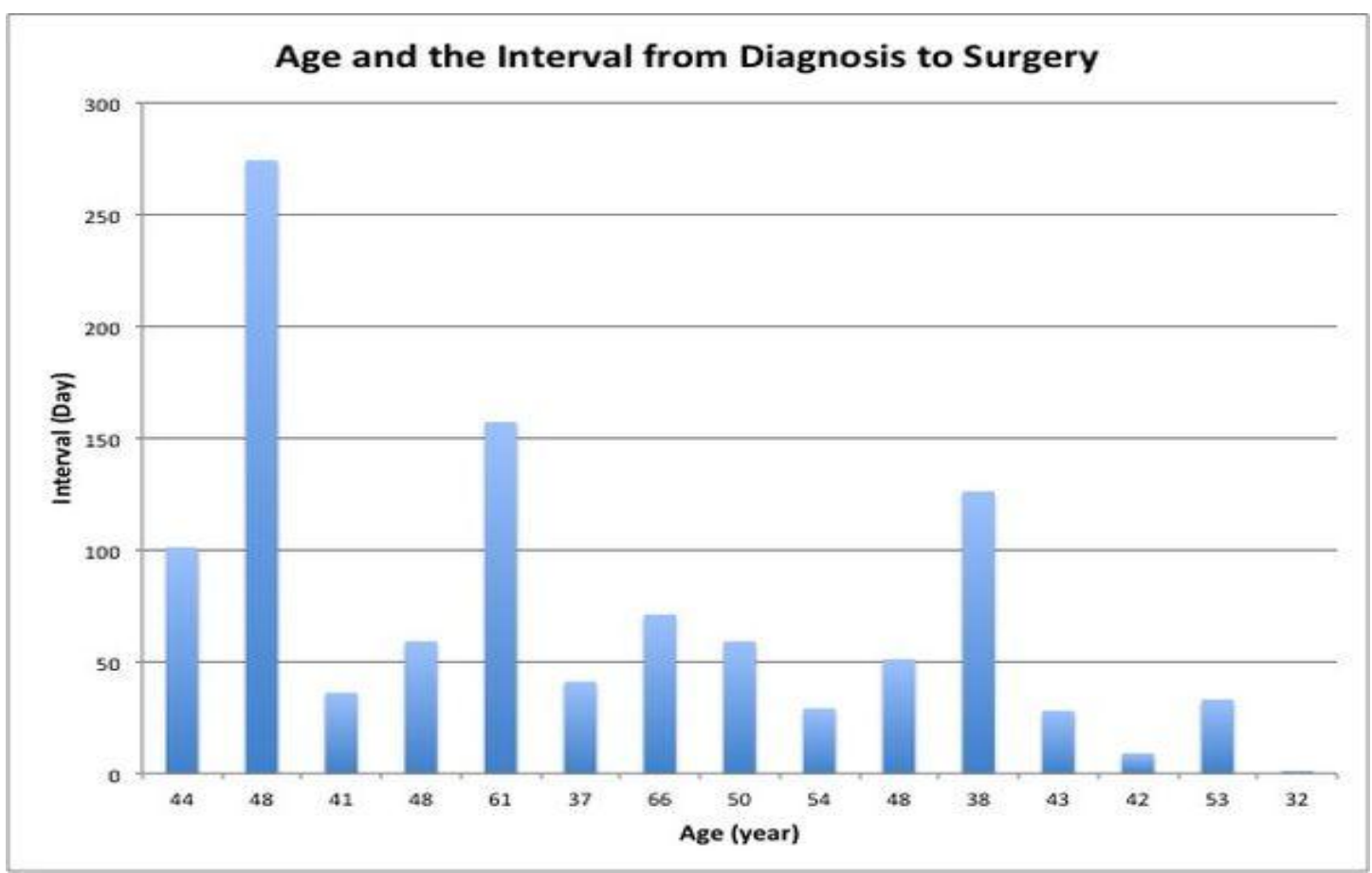

Fig.1:- The interval from the diagnosis to surgery and the patients' age.

\section{Oncoplastic Reduction Techniques}

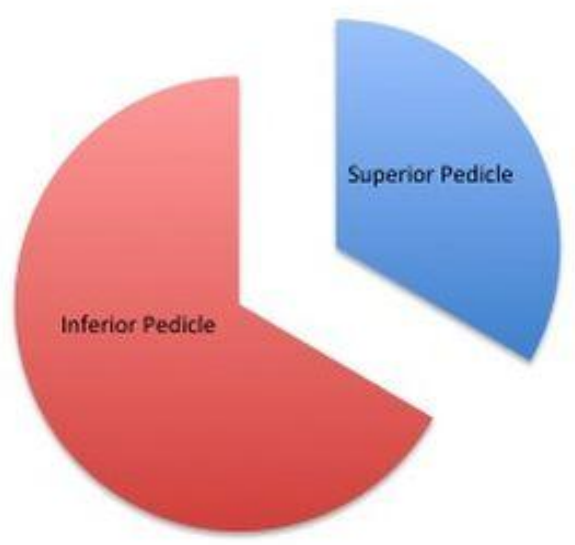

Fig 2:- The different oncoplastic reduction techniques. 


\section{Safety of Oncoplastic Reduction Surgery}

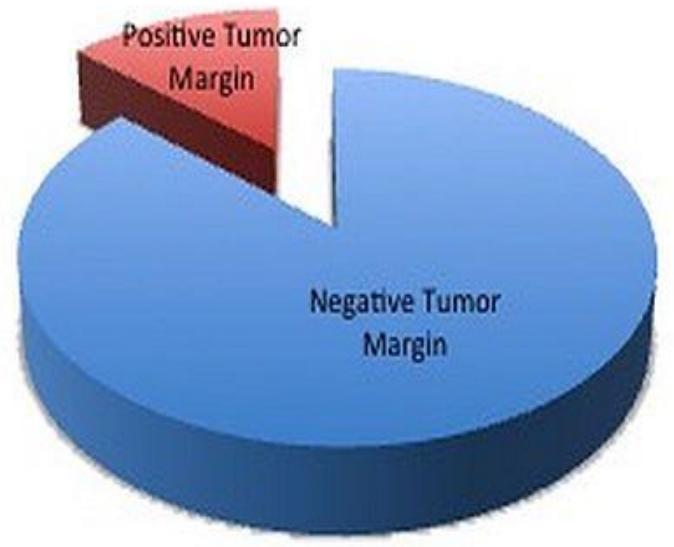

Fig 3:- The safety of oncoplastic reduction surgery.
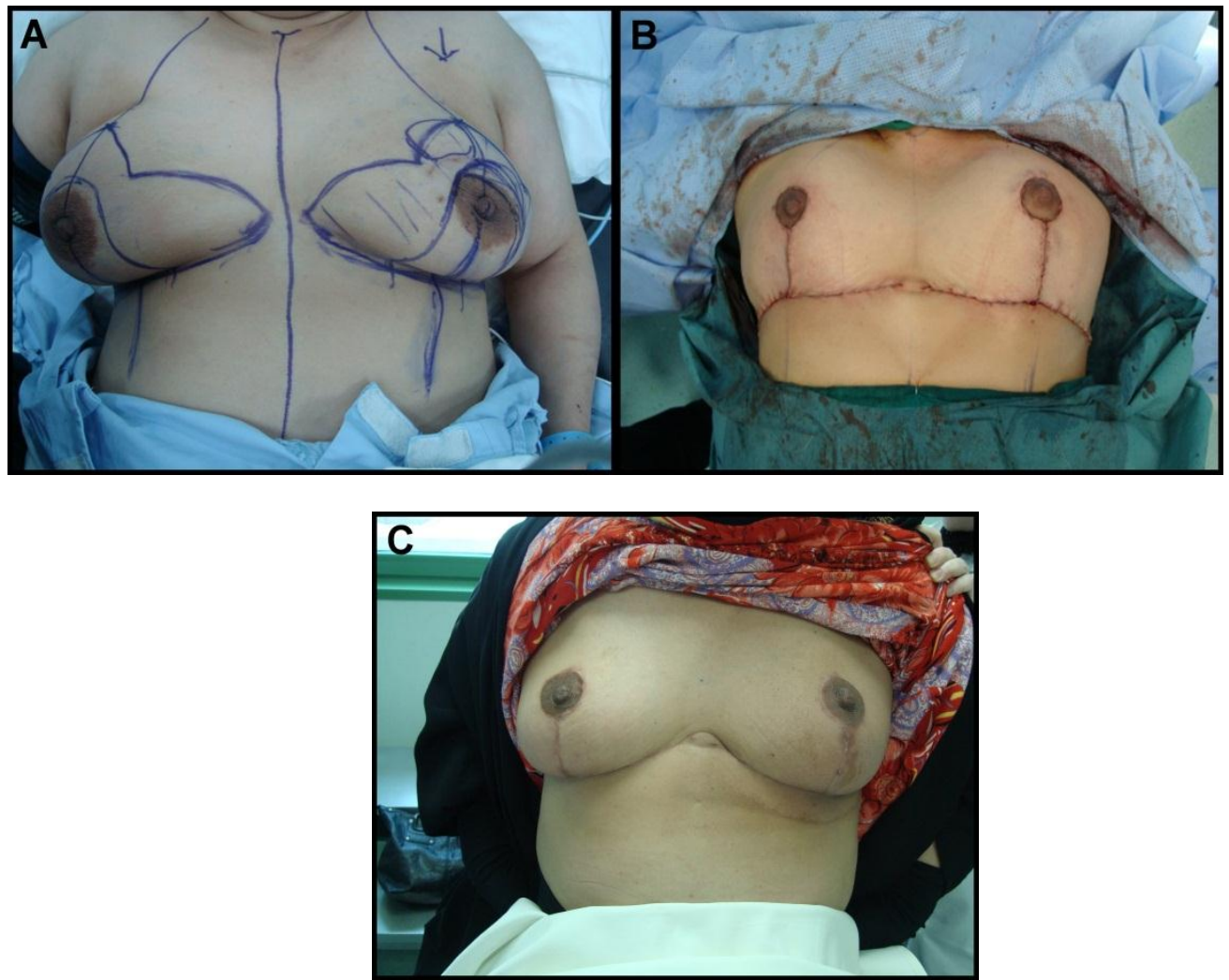

Fig 4:- Inferior Pedicle Technique. (A) Preoperative marking and planning of patient with left breast cancer. (B) Immediate Postoperative result after oncoplastic breast reduction showing location of scars. (C) Three-month postoperative appearance with good outcome. 


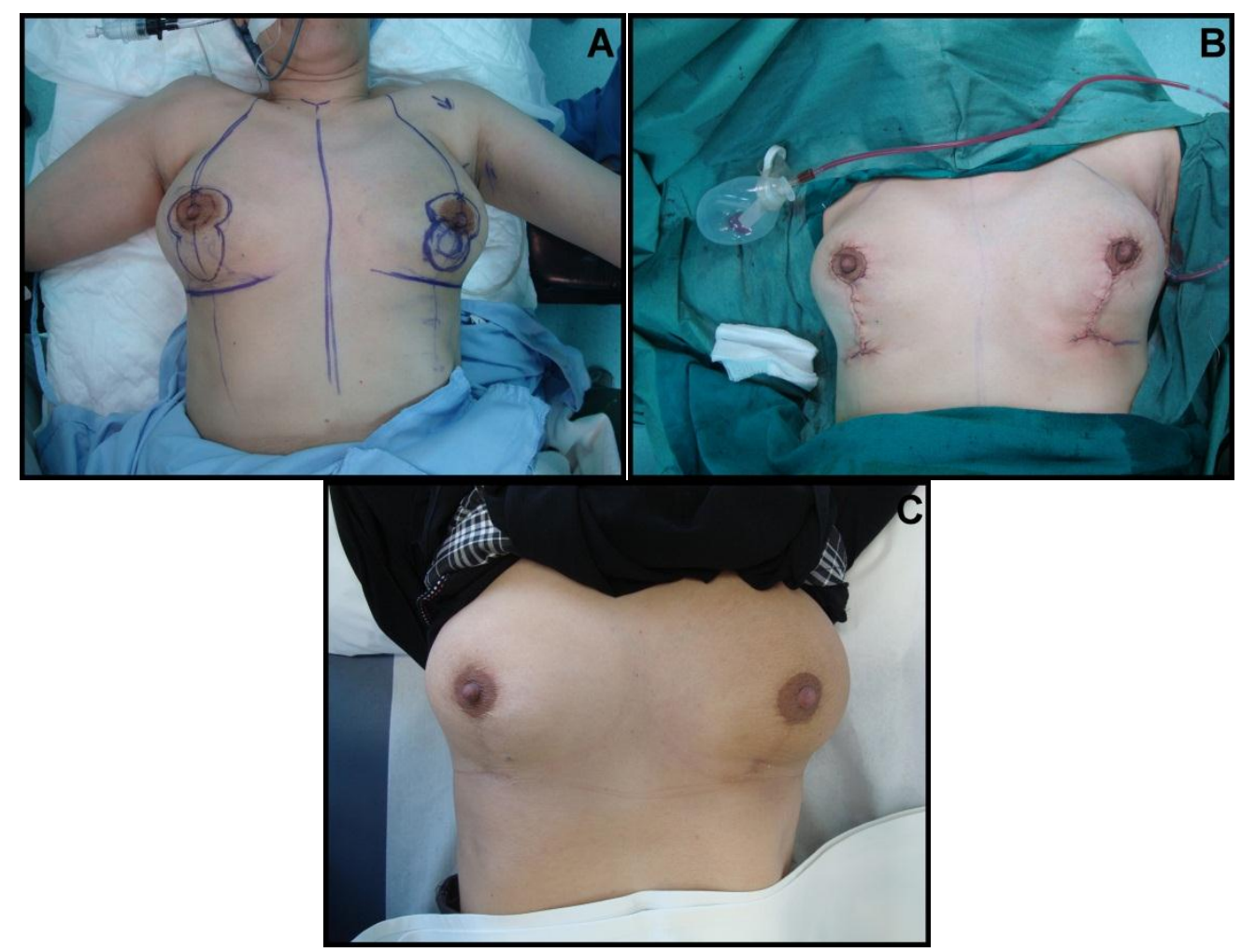

Fig 5:- Superior Pedicle Technique. (A) Preoperative marking and planning of patient with left breast cancer. (B) Immediate Postoperative result after oncoplastic breast reduction showing location of scars. (C) Three-month postoperative appearance with good outcome.

Table 1:- Baseline characteristics of oncoplastic breast reduction patient.

\begin{tabular}{|c|c|}
\hline Characteristics & N (\%) \\
\hline Age, years (mean \pm SD) & $(47 \pm 9.1)$ \\
\hline Interval Dx to ORM, days, (min,max) & $(1,274)$ \\
\hline Breast Cancer & \\
\hline Right & $10(67)$ \\
\hline Left & $5(33)$ \\
\hline IDC & $12(80)$ \\
\hline DCIS & $2(13)$ \\
\hline ILC & $1(7)$ \\
Grade I & $2(13)$ \\
Grade II & $3(20)$ \\
Grade III & $10(67)$ \\
TNM Stage & \\
T1N0M0 & $1(7)$ \\
T1cN1M0 & $1(7)$ \\
ypT1N0Mx & $5(33)$ \\
T2N0M0 & $2(13)$ \\
T2N1M0 & $2(13)$ \\
\hline Tumor size, mm (mean \pm SD) & $(26.41 \pm 17.15)$ \\
Sentinel Lymph Node & \\
Right & $9(60)$ \\
Left & $4(26.7)$ \\
Positive & $2(13.3)$ \\
Negative & $13(86.7)$ \\
\hline Dx: Diagnosis, DCIS: Ductal Carcinoma In Situ , IDC: Invasive Ductal Carcinoma , ILC: Invasive Lobular
\end{tabular}


Carcinoma ,Min: Minimum, Max: Maximum, mm:Millemeter, ORM: Oncoplastic Redcution Mammoplasty.

Table 2:- History characteristics of oncoplastic breast reduction patient.

\begin{tabular}{|c|c|}
\hline Characteristics & N (\%) \\
\hline Age of Menarche, years (mean \pm SD) & $(12.71 \pm 1.85)$ \\
\hline Age at First Birth, years (mean \pm SD) & $(21.71 \pm 3.87)$ \\
\hline Family History & $5(33.3)$ \\
\hline Positive & $9(60)$ \\
\hline Negative & $1(6.7)$ \\
\hline Unknown & \\
\hline Breast Feeding & $12(80)$ \\
Yes & $1(6.7)$ \\
No & $2(13.3)$ \\
Unknown & $11(73.3)$ \\
ER & $9(60)$ \\
PR & $5(33.3)$ \\
Her2 & \\
Positive & $4(26.7)$ \\
ER & $6(40)$ \\
PR & $10(66.7)$ \\
Her2 & $2(13.3)$ \\
Negative Molecular Marker & $9(60)$ \\
Use of OCP & $4(26.7)$ \\
Yes & $2(13.3)$ \\
No & \\
Unknown & \\
Positive & \\
\hline ER: Estrogen Receptor, Her2: Human Epidermal Growth Factor Receptor 2, OCP: Oral Contraceptive Pill, \\
\hline
\end{tabular}

\section{References:-}

1. Hunt Kelly K, Robertson John F.R. The Breast. In: Brunicardi F Charles et al. Schwartz's principles of surgery. 10th Ed. New York: McGraw-Hill Education; 2015. 497- 564.

2. Gabka CJ, Maiwald G, Baumeister RG. Expanding the indications spectrum for breast saving therapy of breast carcinoma by oncoplastic operations. Langenbecks Arch Chir Suppl Kongressbd 1997 114: 1224-7. [Article in German]

3. Baildam,A.D. Oncoplastic surgery for breast cancer. Br J Surg 2008 95: 4-5.

4. Lucy Mansfield, Avi Agrawal, and Ramsey I. Cutress. Oncoplastic breast conserving surgery. Gland Surg 2013 2: 158-62.

5. Morrow M. Minimally invasive surgery for breast cancer. BMJ. 2009;338:b557.

6. Chakravorty A, Shrestha AK, Sanmugalingam N, et al. How safe is oncoplastic breast conservation? Comparative analysis with standard breast conserving surgery. Eur J Surg Oncol. 2012 38:395-8.

7. Fitoussi AD, Berry M, Fama F, et al. Oncoplastic breast surgery for cancer: analysis of 540 consecutive cases [outcomes article]. Plast Reconstr Surg.. 2010;125(2):454-62.

8. Goffman TE, Schneider H, Hay K, Elkins DE, Schnarrs RA, Carman C. Cosmesis with bilateral mammoreduction for conservative breast cancer treatment. Breast J.2005;11(3):195-8.

9. Emiroglu M, Salimoglu S, Karaali C, et al. Oncoplastic reduction mammoplasty for breast cancer in women with macromastia: Oncological long-term outcomes. Asian J Surg. 2015.

10. De La Cruz L, Blankenship SA, Chatterjee A, et al. Outcomes After Oncoplastic Breast-Conserving Surgery in Breast Cancer Patients: A Systematic Literature Review. Ann Surg Oncol. 2016;23(10):3247-58.

11. Kronowitz SJ, Hunt KK, Kuerer HM, et al. Practical guidelines for repair of partial mastectomy defects using the breast reduction technique in patients undergoing breast conservation therapy. Plast Reconstr Surg. 2007 120: 1755-6. 
12. Kaur N, Petit J-Y, Rietjens M, et al. Comparative study of surgical margins in oncoplastic surgery and quadrantectomy in breast cancer. Ann Surg Oncol 2005;12(7):1-7.

13. Losken A, Dugal CS, Styblo TM, Carlson GW. A meta-analysis comparing breast conservation therapy alone to the oncoplastic technique. Ann Plast Surg. 2014;72(2):145-9.

14. Meretoja TJ, Svarvar C, Jahkola TA. Outcome of oncoplastic breast surgery in 90 prospective patients. Am J Surg 2010;200(2):224-228.

15. McCulley S, Macmillan R. Therapeutic mammaplasty - analysis of 50 consecutive cases. Br J Plast Surg. 2005;58(7):902-7.

16. Brinton LA, Persson I, Boice JD Jr, McLaughlin JK, Fraumeni JF Jr. Breast cancer risk in relation to amount of tissue removed during breast reduction operations in Sweden. Cancer 2001;91(3): 478-483.

17. Fernando IN, Ford HT, Powles TJ, et al. Factors affecting acute skin toxicity in patients having breast irradiation after conservative surgery: a prospective study of treatment practice at the Royal Marsden Hospital. Clin Oncol (R Coll Radiol). 1996 Dec 31;8(4):226-33.

18. Iwuchukwu OC, Harvey JR, Dordea M, Critchley AC, Drew PJ. The role of oncoplastic therapeutic mammoplasty in breast cancer surgery—a review. Surg Oncol 2012;21(2):133-141.

19. Rose JF, Colen JS, Ellsworth WAt. Reduction and Mastopexy Techniques for Optimal Results in Oncoplastic Breast Reconstruction. Semin Plast Surg. 2015;29(2):102-9.

20. Pusic AL, Klassen AF, Cano SJ. Use of the BREAST-Q in clinical outcomes research. Plast Reconstr Surg. 2012 Jan 1;129(1):166e-7e.

21. Chang E, Johnson N, Webber B, et al. Bilateral reduction mammoplasty in combination with lumpectomy for treatment of breast cancer in patients with macromastia. Am J Surg. 2004;187(5):647-51.

22. Cochrane RA, Valasiadou P, Wilson AR, Al-Ghazal SK, Macmillan RD. Cosmesis and satisfaction after breastconserving surgery correlates with the percentage of breast volume excised. Br J Surg. 2003 Dec 1;90(12):1505-9. 